\title{
Study of the Prevalence of Hypertension and Complications of Hypertensive Disorders in Pregnancy
}

\author{
Shahla Khosravi ${ }^{1}$, Soheila Dabiran ${ }^{2 *}$, M. Lotfi ${ }^{3}$, Mohammad Asnavandy ${ }^{2}$ \\ ${ }^{1}$ Reproductive Health and Community Medicine, Faculty of Medical Sciences, Tehran University of Medical \\ Sciences, Tehran, Iran \\ ${ }^{2}$ Department of Community Medicine, Tehran University of Medical Sciences, Tehran, Iran \\ ${ }^{3}$ Islamic Azad University of Varamin, Varamin, Iran \\ Email: ${ }^{*}$ dabirans@tums.ac.ir
}

Received 21 September 2014; revised 22 October 2014; accepted 18 November 2014

Copyright (C) 2014 by authors and Scientific Research Publishing Inc.

This work is licensed under the Creative Commons Attribution International License (CC BY). http://creativecommons.org/licenses/by/4.0/

\section{Open Access}

\section{Abstract}

Introduction: Hypertension is one of the common problems associated with pregnancy that may be followed by eclampsia, acute renal failure, maternal death, premature delivery, intra-uterine growth restriction and other. This study was conducted to determine the results of pregnancies associated with hypertension in patients visiting in the Delivery Ward of Valiasr Hospital. Methods: A descriptive study was conducted on all the patients admitted to the aforementioned department and who possessed the inclusion criteria for hypertensive pregnancy. Results: Among the 1694 delivery cases examined, 173 cases had hypertension (9.8\%). Among these, 75 (45\%) had gestational hypertension; $24(14.8 \%)$ had preeclampsia-eclampsia; $30(18 \%)$ had preeclampsia superimposed on chronic hypertension; 21 (13.5\%) cases had chronic hypertension; and $13(8 \%)$ had pregnancy-aggravated chronic hypertension. Ninety-six point three percent $(96.3 \%)$ had a systolic blood pressure (BP) of $140-190 \mathrm{mmHg}$, and 3.7\% had a systolic BP greater than $190 \mathrm{mmHg}$. Whereas $61.1 \%$ of diastolic blood pressure $90-110 \mathrm{mmHg}$ and $38.9 \%$ of the mothers had diastolic BP greater than $110 \mathrm{mmHg}$. The HELLP (Hemolysis, Elevated Liver enzymes \& Low Platelet count) syndrome was present in $4.9 \%$ of cases; $52.6 \%$ experienced premature delivery; $7.4 \%$ had IUFD (intra uterine fetal death); 9.9\% had IUGR (intrauterine growth retardation); and $17.3 \%$ had LBW babies. Conclusions: Based on our results, hypertensive mothers who are younger and have lower weight babies at birth experience more perinatal complications. The unpleasant effects of hypertension in pregnancy warrant the need for training, routine prenatal care, the early detection and treatment of hypertension at younger ages of pregnancy, and follow-up after delivery.

"Corresponding author.

How to cite this paper: Khosravi, S., Dabiran, S., Lotfi, M. and Asnavandy, M. (2014) Study of the Prevalence of Hypertension and Complications of Hypertensive Disorders in Pregnancy. Open Journal of Preventive Medicine, 4, 860-867. 


\section{Keywords}

\section{Hypertension, Pregnancy Induced Hypertension, Preeclampsia, Eclampsia, Chronic Hypertension}

\section{Background}

Hypertensive disorders of pregnancy are among the leading causes of maternal and perinatal deaths in developing countries, and many studies have been conducted in this field [1]. Hypertension is a common medical problem that affects $20 \%$ - 30\% of the adult population and more than $5 \%-8 \%$ of all pregnancies in the world [2] [3]. Hypertensive disorders of pregnancy rank high among the causes of maternal mortality and morbidity. Hypertension in pregnancy is defined as a systolic BP of $140 \mathrm{mmHg}$ and higher, and a diastolic BP of $90 \mathrm{mmHg}$ and higher. Being the second leading cause of maternal death in the USA, almost $15 \%$ of maternal deaths are related to hypertension (HTN). Severe HTN raises the risk of heart attacks, cardiac failure, cerebrovascular accidents and renal failure in the mothers. The fetuses of hypertensive mothers are also at increased risks, such as: inappropriate placental oxygen transfer, IUGR, premature delivery, placental abruption, stillbirth, and neonatal death [2]. The hypertensive disorders of pregnancy affect 5\% - 22\% of all pregnancies. Hypertension, bleeding and infection are the triad of lethality that greatly contributes to maternal mortality and morbidity [4]-[7].

The American College of Obstetricians and Gynecologists (ACOG) has classified pregnancy induced hypertension (PIH) into four groups of disorders: gestational hypertension, where resting BP is 140/90 mmHg or higher after the $20^{\text {th }}$ week of gestation; chronic hypertension, that exists before pregnancy or begins in the first 20 weeks of gestation; preeclampsia (raised BP and edema or proteinuria)/eclampsia (preeclampsia and seizures); and preeclampsia superimposed on chronic hypertension [3] [4]. In spite of the high incidence and outcomes of hypertensive disorders of pregnancy, their pathogenesis, clinical manifestations and clinical courses greatly vary and at times complicate the diagnosis [8]. Decades of extensive research on the subject have failed to explain its onset or aggravation, and it is still the most significant unsolved problem in midwifery. Among the theories proposed so far, the most important is the immunologic one. Based on this theory, an immunologic disorder leads to an unnatural implantation and secretion of substances that activate vascular endothelial cells, or damage them, leading to vascular constriction and eventually a raised BP.

PIH is more commonly seen in nulliparous women, and older women (owing to the risk of chronic HTN) are at greater risk of preeclampsia being superimposed. Evidence shows that discrete pathophysiological changes begin from the moment fertilization takes place. And if delivery does not take place these changes lead to the involvement of multiple organs and present with dangerous clinical signs in both the mother and fetus [4]. Pregnancies induced with hypertension are associated with increased risks of serious outcomes such as premature delivery, IUGR, perinatal mortality \& morbidity, acute renal failure (ARF), acute hepatic failure, bleeding at the time of delivery and postpartum bleeding, maternal mortality \& morbidity following harms such as HELLP, disseminated intravascular coagulation (DIC) and seizures [2]-[4] [9]. The hazards of these outcomes depend on its severity, gestational age (GA) at the onset of HTN, and GA at the time of delivery. According to literature, women who are affected with PIH before their $37^{\text {th }}$ week of gestation have poorer perinatal results as compared to women who are affected at term. Moreover, IUGR and placental abruption are more common in preterm deliveries. Mothers who have had a history of preeclampsia (17.9\%) are at a greater risk than nulliparous women $(5.3 \%)(\mathrm{p}<0.00001)$ [9]. Hypertensive mothers usually give birth to preterm babies, who in turn will most likely need Neonatal Intensive Care Unit (NICU) care as a result of their IUGR and low birth weight (LBW) [10].

The prevalence of preeclampsia has been reported at 5\%, but it is influenced by parity, race, ethnicity, environmental factors, socio-economic status, multiple pregnancies, maternal obesity etc. Hence different statistical results have been obtained in research. This study aimed to investigate the consequences of high blood pressure in pregnancy.

\section{Methods}

A cross-sectional descriptive study was conducted in Imam Khomeini Hospital (IKH), a teaching hospital affiliated with Tehran University of Medical Sciences. This hospital is also considered a tertiary care center. All 
hypertensive pregnant mothers (with a BP of 140/90 mmHg or more) who had visited the hospital for delivery during one year went under study, regardless of when their BP had risen. Among the 1694 cases of delivery 173 had hypertension (9.8\%), 11 of which who were excluded because of incomplete data. The maternal variables studied were: age, gestational age, number of deliveries, status of mother's BP, type of delivery, time of occurrence of HELLP based on lab findings and doctor's diagnosis, and maternal death. Neonatal variables included: weight at birth, first minute and fifth minute Apgar scores, IUGR, IUFD and neonatal death. Data were extracted upon referring to mothers' and neonates' existing files, and gathered by completing the study's data collection questionnaire.

Sampling: All hypertensive patients visiting the delivery ward were studied as a census. The reason for choosing IKH was its NICU ward and its proximity to the delivery ward and hence the possibility to follow-up the newborns. The inclusion criteria were: delivery at IKH, existence of adequate data in the mother's and neonate's files, and existence of HTN (with respect to our set criteria).

In our study hypertension was considered as a systolic BP of $140 \mathrm{mmHg}$ and higher and a diastolic BP of 90 mmHg and higher. Based on the signs \& symptoms of disease and a history of HTN before pregnancy, the patients were classified into groups of "gestational hypertension", "chronic HTN", "pregnancy-aggravated chronic HTN", "preeclampsia-eclampsia” and "preeclampsia superimposed on chronic hypertension". The data existing in the files have been registered upon a physician's diagnosis. Thenceforward the data were fed into a designed database and analyzed by SPSS software.

\section{Results}

Among the 1694 mothers who had delivered in the hospital 173 had HTN (9.8\%), 11 of which were excluded because of incomplete data, hence leaving us with 162 cases. Most mothers fell in the 21 - 30 years age-group (55.6\%). Nine point four percent (9.4\%) were aged under 20 and 32.3\% were aged over 30 years. 38.6\% were nulliparous and only $3.1 \%$ had over 5 pregnancies. The study population's demographic data are displayed in Table 1.

Table 1. Demographic characteristics of the population and their newborn infants of mothers with hypertension.

\begin{tabular}{cccc}
\hline Variables & & Number & Percent (\%) \\
\hline \multirow{2}{*}{ Maternal age } & $<20$ & 15 & 12.2 \\
& $21-30$ & 90 & 55.6 \\
& $>30$ & 57 & 32.2 \\
Parity & 1 & 52 & 32 \\
& 2 & 44 & 27.1 \\
Gestational & $3-5$ & 59 & 36.4 \\
& $20-29$ & 6 & 3.7 \\
Type of delivery & $30-36$ & 12 & 7.4 \\
& $\leq 37$ & 93 & 57.4 \\
Birth weight & NVD & 57 & 35.1 \\
& Cesarean section & 53 & 32.7 \\
First minute Apgar score & $700-1499$ & 109 & 67.3 \\
Birth height & $1500-2400$ & 9 & 5.5 \\
\hline
\end{tabular}

Fetuses born dead, weight, and Apgar score has not been calculated. 
More than half the mothers' (52.6\%) GA at delivery (spontaneous, induced and/or elective section) was under 37 weeks and gave birth to preterm babies. Among these 7.4\% (12) babies were born at 20 - 28 weeks GA, where viability is very poor. Among the latter $28.1 \%$ were LBW babies or had IUGR.

Eighteen point three percent (18.3\%) of the newborns (28 cases) were LBW babies, weighing less than $2500 \mathrm{~g}$. Among these, $32.1 \%$ (9 newborns) weighed less than 1500 g, i.e. were very LBW babies. The stillborn cases were however not measured for weight and height. Only two babies weighed $3500 \mathrm{~g}$ or higher in this study. Fifty-five percent (90 newborns) measured less than $50 \mathrm{~cm}$ in height, $8 \%$ (13) of which measured less than $40 \mathrm{~cm}$. The stillborn cases or those who had early death were either not measured and/or had not been registered anywhere.

The frequency distribution of systolic and diastolic BP in hypertensive mothers is illustrated in Table 2. According to the results, the percentage of very high diastolic BP-which is an indicator of the severity of disease - was more than the percentage of very high systolic BP in these individuals.

In hypertensive mothers, 7.4\% of newborns (12 cases) were born at a GA of 20 - 28 weeks, $45.2 \%$ (73) cases were born at 29 - 36 weeks GA, and 37.4\% (77 cases) were born at 37 - 42 weeks GA. Hence the highest percentage was of those born at 29 - 36 weeks GA. Beginning with the third semester, PIH had posed as a serious threat and had led to the termination of pregnancy.

Upon examining the first minute Apgar score, 2.5\% (4) of newborns scored lower than 4, 19.8\% (32) scored between 5 - 6, and 74.4\% (121) scored between 7 - 10. The fifth minute Apgar score was lower than 7 in $1.9 \%$ (3) cases, and 7 or more in $98.1 \%$ cases.

Table 3 shows the frequency distribution of different types of PIH.

The maternal and fetal complications of HTN raise the possibility of perinatal and neonatal mortality and morbidity. Preterm delivery was the most prevalent among such outcomes. Some of these cases were the result of induction or elective termination of pregnancy as the most important measures in controlling and managing hypertensive disorders and reducing the risks to mother and fetus. Table 4 shows the frequency of these complications.

Table 2. Distribution of systolic and diastolic blood pressure values.

\begin{tabular}{cccccc}
\hline Diastolic blood pressure $(\mathrm{mmHg})$ & Number & Percent & Systolic blood pressure $(\mathrm{mmHg})$ & Number & Percent \\
\hline $90-110$ & 99 & 61.1 & $140-190$ & 156 & 96.3 \\
$110-140$ & 63 & 38.9 & $191-230$ & 6 & 3.7 \\
Total & 162 & 100 & Total & 162 & 100 \\
\hline
\end{tabular}

Table 3. Prevalence types of hypertensive disorders in the study population.

\begin{tabular}{ccc} 
Disorders & Number & Percent (\%) \\
\hline Gestational hypertension & 74 & 45 \\
Preeclampsia-eclampsia & 24 & 14.8 \\
Preeclampsia superimposed on chronic hypertension & 30 & 18 \\
Chronic hypertension & 21 & 13.5 \\
Pregnancy-aggravated chronic hypertension & 13 & 102 \\
Total & 162 & 100 \\
\hline
\end{tabular}

Table 4. Prevalence of maternal and fetal outcomes in the study population.

\begin{tabular}{|c|c|c|}
\hline Complications & Number & Percent (\%) \\
\hline HELLPs syndrome & 8 & 4.9 \\
\hline Preterm labor & 85 & 52.6 \\
\hline Cesarean section & 109 & 67.3 \\
\hline LBW & 28 & 17.3 \\
\hline IUGR & 16 & 9.8 \\
\hline IUFD & 9 & 5.5 \\
\hline Infant death & 10 & 6.1 \\
\hline Mother death & 0 & 0 \\
\hline
\end{tabular}


Thirty-two percent (53) mothers had natural vaginal deliveries (NVD), and 67.3\% went under cesarean section. Termination of pregnancy was done by induction in $38.3 \%$ (62) cases, $5.6 \%$ of which ended up in cesarean. Elective cesarean was performed in $60.5 \%$ (98) cases.

\section{Discussion}

Maternal and fetal complications increase with an increase in PIH, but appropriate maternal and perinatal care can prevent dangerous outcomes such as eclampsia and maternal death.

In a population-based study, Roberts et al. (2005) examined hypertensive pregnancy disorders in 250,173 pregnant women and their newborns in Sydney-Australia. On the whole, 9.8\% of the mothers had PIH disorders; $6 \%$ of these had chronic HTN, $4.2 \%$ were affected with preeclampsia, $0.3 \%$ had preeclampsia superimposed on chronic HTN, and $4.3 \%$ had gestational HTN. Mothers affected with hypertensive disorders were more exposed to maternal mortality and morbidities as compared to those who were not affected [10]. Another study conducted in Nigeria on 2393 deliveries found 127 (5.3\%) cases affected with PIH disorders. Gestational HTN, preeclampsia superimposed on chronic HTN and preeclampsia/eclampsia were observed in $26.2 \%, 19.7 \%$ and $54.1 \%$ cases, respectively. All the cases that had had chronic HTN before pregnancy had experienced superimposed preeclampsia or eclampsia during pregnancy [11].

In our study, 1694 mothers visiting IKH’s delivery department during one year were studied. 173 cases had HTN (9.8\%). Among these, $60.5 \%$ had preeclampsia-eclampsia, 30 (18\%) had preeclampsia superimposed on chronic HTN, 21 (13.5\%) cases had chronic HTN and 13 (8\%) had pregnancy-aggravated chronic HTN.

A study in Madrid (2004) examined the outcomes of chronic HTN in the second half of pregnancy in 154 mothers. Seventy-two percent (72\%) had had chronic HTN before pregnancy and the remainder had been affected with it during the first half of pregnancy. Seventy-eight percent (78\%) had experienced aggravation of HTN during pregnancy. Their mean gestational age was $34 \pm 4.6$ weeks, and most of them had undergone cesarean [12].

Dr. Jain of Chicago's Illinois University conducted a study on 109,428 deliveries. During the study 8019 mothers had PIH, among which $74.5 \%$ had preeclampsia and $25.5 \%$ had chronic HTN. Hypertension was associated with unpleasant outcomes in pregnancy, such that in this study chronic HTN was associated with preterm delivery and IUGR more as compared to PIH alone [13]. A third of women who have chronic HTN are affected with preeclampsia in pregnancy and this phenomenon usually takes place between 26 - 34 weeks GA [2]. In our study, $39.5 \%$ of the mothers had chronic HTN prior to pregnancy, $18 \%$ of which were affected with preeclampsia. Eclampsia superimposed on chronic HTN doubles the risk of IUGR and preterm delivery [2] [12].

The risk of premature birth in mothers with PIH is approximately 25\% - 30\% [12]. Preeclampsia usually occurs in the $32^{\text {nd }}$ week and after. Ninety-four percent (94\%) of the cases Saleh et al. (2003) followed had been affected with PIH at the $32^{\text {nd }}$ week and onwards [14]. Whereas, preeclampsia superimposed on chronic HTN usually occurs in the 26 - 33 weeks GA, and raises the risk of premature birth and its outcomes [2]. In Bozhinova et al.'s study (2004) the severe forms of preeclampsia/eclampsia were seen to begin in the 25 - 30 weeks of pregnancy in $60 \%$ of cases, whereas the semi-severe forms of the disease began during 31 - 36 weeks in 50\% of cases [15]. Among the 760 mothers kept under observation in Moodley et al.'s study (1999) in South Africa, $46.3 \%$ had preterm deliveries [16]. In our study however, $52.6 \%$ of the pregnancies ended up in preterm deliveries, 7.4\% of which was terminated between 20 - 29 weeks. However, some of these deliveries were the result of induction. The definitive treatment of preeclampsia is termination of pregnancy, which is done in spite of the prematurity of the baby [2].

A case control study in Buenos Aires (1999) hospitals examined the type of deliveries and found that the number of cesarean (cases with PIH) were 3.8 times the controls, concluding that PIH was clearly associated with increased cesarean [17]. The greater the severity of HTN the more are the complications associated with it during pregnancy, and hence, the greater the possibility of pregnancy termination [1] [13]. Granguly et al. reported a 34.3\% rate of cesarean section among hypertensive mothers [18]. Zibaeenezhad et al. reported a 45.8\% rate [19]. In our study, $67.3 \%$ of pregnancies had been terminated by cesarean. Although the rate of cesarean is high among PIH mothers, the higher figures in our study—as opposed to others—is because the overall rate of cesarean in our country is many times the ideal figure. Like many other countries, Iran too has witnessed an alarming increase in the cesarean rate; $48 \%$ of all deliveries in 2009 were performed through cesarean [20]. This is in spite the fact that the World Health Organization (WHO) has announced the normal cesarean rate at $10 \%$ - 
$15 \%$ of all deliveries.

Induction of delivery is used to terminate pregnancy in hypertensive mothers. In our study 38.3\% (62) mothers were induced to deliver, $5.6 \%$ of which ended up in cesarean. While in Yadav's study where 250 cases (PIH women) and 400 controls were observed, $52.8 \%$ and $3.25 \%$ of cases and controls, respectively, were induced to deliver. Among those induced to deliver, $14.8 \%$ and $3.5 \%$ of cases and controls, respectively, underwent cesarean [21].

One of the serious complications associated with severe preeclampsia is the HELLP syndrome. This syndrome is a group of symptoms including hemolysis, elevated liver enzymes, and low platelet count. There were 4.9\% cases of HELLP syndrome in our study, whereas Vigil observed the HELLP syndrome, ARF, pulmonary edema and hypertensive-induced encephalopathy following delivery in $8.4 \%, 8.4 \%, 3.9 \%, 1.3 \%$ and $1.3 \%$ of cases [12]. Moodley reported HELLP syndrome at 3.2\% [16], and Arif Khan reported it at 3.8\% in Agha Khan's Hospital. In Ben et al.'s study the frequency of this syndrome in mothers affected with severe preeclampsia and/or eclampsia was reported as 9.7\% (167 cases), 14 of which occurred before and 2 of which occurred after delivery. Nine cases had acquired it before the $32^{\text {nd }}$ week of pregnancy [21]. Osmanagaoglu (2004) found a significant association between the occurrence of HELLP syndrome and maternal mortality and morbidity [22]. However, we found no case of maternal mortality in our study, although Moodley reported it as $0.8 \%$.

The mean birth weight of babies born to hypertensive mothers is lower than normal [9]. The mean birth weight of babies born to chronically hypertensive mothers is also lower than pregnancy-induced hypertensive mothers (with a difference of $184 \mathrm{~g}$ ) [12]. In our study $17.3 \%$ of the babies born to hypertensive mothers had birth weights less than $2500 \mathrm{~g}$, which is greater than the normal population. Out of this $17.3 \%$, 5.5\% weighed less than 1500 g. The study conducted by Moodley in South Africa (1999) divided the 322 nulliparous that consisted of 161 hypertensive cases and 161 controls into two groups: a) those with proteinuria, and b) those without proteinuria. The mean birth weight in the control group was $3.02 \mathrm{~kg}$. It was $2.4 \mathrm{~kg}$ and $2.8 \mathrm{~kg}$ in groups "a" and "b" respectively. There were 9 cases of perinatal death, all of which were observed in the hypertensive group [16].

Preeclampsia superimposed on chronic hypertension usually occurs between weeks 26 - 34, and doubles the risk of preterm delivery and IUGR [13]. A study conducted by Fernandes on the Italian Hospital in Buenos Aires showed that SGA (small for gestational age) babies were born 7.08 times more to mothers with PIH, than in the control group [17]. The frequency of IUGR was $9.8 \%$ in our study, and $6.6 \%$ in Moodley and others' studies. The higher rate of IUGR in our study is probably a consequence of economic conditions and poor nutrition during pregnancy. The greatest rate of IUGR was observed in the 21 - 30 years old group (62\%).

Elevation of BP in the mother increases the risk of perinatal mortality and morbidity. There were $9(5.6 \%)$ such cases (IUFD and neonatal death) among the 161 hypertensive nulliparous observed in Moodley's study [16]. Jain's study reported $4.8 \%$ perinatal mortality and morbidity in PIH mothers, as opposed to $2.9 \%$ in chronically hypertensive mothers [12]. We estimated IUFD at 5.5\% and neonatal death at $6.1 \%$. We had 9 cases of IUFD and/or neonatal deaths, all of which took place in hypertensive mothers who had proteinuria. Yadav also reported higher IUFD rates in hypertensive mothers, where it was $4.8 \%$, as opposed to $0.25 \%$ in the control group. Perinatal deaths were observed in $14.8 \%$ of hypertensive mothers, as opposed to $1 \%$ in the control group, hence necessitating special neonatal care in the hypertensive group [21]. Hadavi et al. studied the causes of death in a two-year study and found PIH to be one of the most common causes of perinatal mortality and morbidity at a rate of $8.9 \%$ [23]. Elsewhere Babaee et al. examined 445 mothers and neonates and looked for the causes of mortality and morbidity in premature babies, and found that $21 \%$ of LBW babies had been born to mothers with PIH [24].

\section{Limitations}

The most important limitation of this study was the availability of information in the hospital files, which did not allow for their further examination.

\section{Conclusion}

Pregnancy-induced hypertension is associated with multiple complications in the mother and baby, and particularly preterm delivery. Complications such as HELLP syndrome can sometimes prove fatal to mother and fetus. 
Therefore, the timely diagnosis of PIH and provision of specialized antenatal maternal care could reduce the impacts of such complications.

\section{References}

[1] Henry, C.S., Biedermann, S.A., Campbell, M.F. and Guntupalli, J.S. (2004) Spectrum of Hypertensive Emergencies in Pregnancy. Critical Care Clinics, 20, 607-712. http://dx.doi.org/10.1016/j.ccc.2004.05.014

[2] De Cherney, A.H., Nathan, L., Laufer, N. and Roman, A.S. (2012) Current Diagnosis \& Treatment: Obstetrics \& Gynecology. 11th Edition, Chapter 26: Hypertension in Pregnancy.

[3] (2000) Report of the National High Blood Pressure Education Program Working Group on High Blood Pressure in Pregnancy. American Journal of Obstetrics and Gynecology, 183, S1-S22. http://dx.doi.org/10.1067/mob.2000.107928

[4] Cunnigham, F.G., Leveno, K., Bloom, S., et al. (2010) Willams Obstetrics. 23rd Edition, McGraw-Hill, Medical Publishing Division, New York.

[5] Vest, A.R. and Cho, L.S. (2012) Hypertension in Pregnancy. Cardiology Clinics, 30, 407-423. http://dx.doi.org/10.1016/j.ccl.2012.04.005

[6] Wagner, S.J., Barac, S. and Garovic, V.D. (2007) Hypertensive Pregnancy Disorders: Current Concepts. Journal of Clinical Hypertension, 9, 560-566. http://dx.doi.org/10.1111/j.1524-6175.2007.06695.x

[7] Henry, C.S., Biedermann, S.A., Campbell, M.F. and Guntupalli, J.S. (2004) Spectrum of Hypertensive Emergencies in Pregnancy. Critical Care Clinics, 20, 697-712. http://dx.doi.org/10.1016/j.ccc.2004.05.014

[8] Hnat, M.D., Sibai, B.M., Caritis, S., Hauth, J., Lindheimer, M.D., MacPherson, C., Van Dorsten, J.P., Landon, M., Miodovnik, M., Paul, R., Meis, P., Thurnau, G. and Dombrowski, M., for the National Institute of Child Health and Human Development Network of Maternal-Fetal Medicine Units (2002) Perinatal Outcome in Women with Recurrent Preeclampsia Compared with Women Who Develop Preeclampsia as Nulliparas. American Journal of Obstetrics \& Gynecology, 20, 422-426. http://dx.doi.org/10.1067/mob.2002.120280

[9] Roberts, C.L., Albert, C.S., Morris, J.M., et al. (2005) Hypertensive Disorders in Pregnancy: A Population-Based Study. Medical Journal of Australia, 182, 332-335.

[10] Familoni, O.B., Adefuye, P.O. and Olunuga, T.O. (2004) Pattern and Factors Affecting the Outcome of Pregnancy in Hypertensive Patients. Journal of the National Medical Association, 96, 1626-1631.

[11] Vigil-De Garcia, P., Montufar-Rueda, C. and Smith, A. (2004) Pregnancy and Severe Chronic Hypertension: Maternal Outcome. Hypertension in Pregnancy, 23, 285-293. http://dx.doi.org/10.1081/PRG-200030315

[12] Jain, L. (1997) Effects of Pregnancy-Induced and Chronic Hypertension of Pregnancy Outcome. Journal of Perinatology, 17, 425-427.

[13] Saleh, M.M. and Selinger, M. (2005) Evaluation of the Role of Day Assessment Unit in the Management of Pregnancy Induced Hypertension. Journal of Obstetrics \& Gynaecology, 25, 651-655. http://dx.doi.org/10.1080/01443610500278345

[14] Bozhinova, S., Poroshanova, V. and Sampat, D. (2004) Delivery and Perinatal Aspects of Pregnant Women with Preeclampsia Eclampsia. Akusherstvo i Ginekologiia, 43, 3-9.

[15] Moodley, J., Mphatsoe, M. and Gouws, E. (1999) Pregnancy Outcome in Primigravidae with Late Onset Hypertensive Disease. East African Medical Journal, 76, 490-494.

[16] Fernández Jonusas, S., Ceriani, C. and Ernadas, J.M. (1999) The Effects of Arterial Hypertension during Pregnancy on Birth Weight, Intrauterine Growth Retardation and Neonatal Evolution. A Matched Case-Control Study. Anales Españoles de Pediatría, 50, 52-56.

[17] Ganguly, S. and Begum, A. (2007) Rate of Caesarean Operation and Complications in Hypertensive Disorders of Pregnancy. The ORION Medical Journal, 27, 463-466.

[18] Zibaeenezhad, M.J., Ghodsi, M., Arab, P. and Gholzom, N. (2010) The Prevalence of Hypertensive Disorders of Pregnancy in Shiraz, Southern Iran. Iranian Cardiovascular Research Journal, 4, 169-172.

[19] Maternal Health Office (2010) Unpublished Data. Ministry of Health and Medical Education. Tehran.

[20] Yadav, S., Saxena, U., Yadav, R. and Gupta, S. (1997) Hypertensive Disorders of Pregnancy. Journal of Indian Medical Association, 95, 548-551.

[21] Ben Letaifa, D., Ben Hamada, S., Salem, N., Ben Jazia, K., Slama, A., Mansali, L. and Jegham, H. (2000) Maternal and Perinatal Morbidity and Mortality Associated with Hellp Syndrome. Annales Françaises d'Anesthésie et de Réanimation, 19, 712-718. http://dx.doi.org/10.1016/S0750-7658(00)00313-0

[22] Osmanagaoglu, M.A., Erdogan, I., Zengin, U. and Bozkaga, H. (2004) Comparison between HELLP Syndrome, 
Chronic Hypertension, and Superimposed Preeclampsia on Chronic Hypertension without HELLP Syndrome. Journal of Perinatal Medicine, 32, 481-485. http://dx.doi.org/10.1515/JPM.2004.132

[23] Hadavi, M., Alidalaky, S., Abedini, M. and Aminzadeh, F. (2009) Factors Affecting Perinatal Mortality in the City of Rafsanjan Medical Centers in 2004-2006. Rafsanjan Medical Sciences Journal, 8, 117-126.

[24] Babaee, Gh., Keshavarz, M., Parsinia, M. and Ashkvari, P. (2008) Evaluation of Effective Factors on Low Birth Weight Neonates’ Mortality Using Path Analysis. Tehran University Medical Journal, 66, 52-56. 
Scientific Research Publishing (SCIRP) is one of the largest Open Access journal publishers. It is currently publishing more than 200 open access, online, peer-reviewed journals covering a wide range of academic disciplines. SCIRP serves the worldwide academic communities and contributes to the progress and application of science with its publication.

Other selected journals from SCIRP are listed as below. Submit your manuscript to us via either submit@scirp.org or Online Submission Portal.
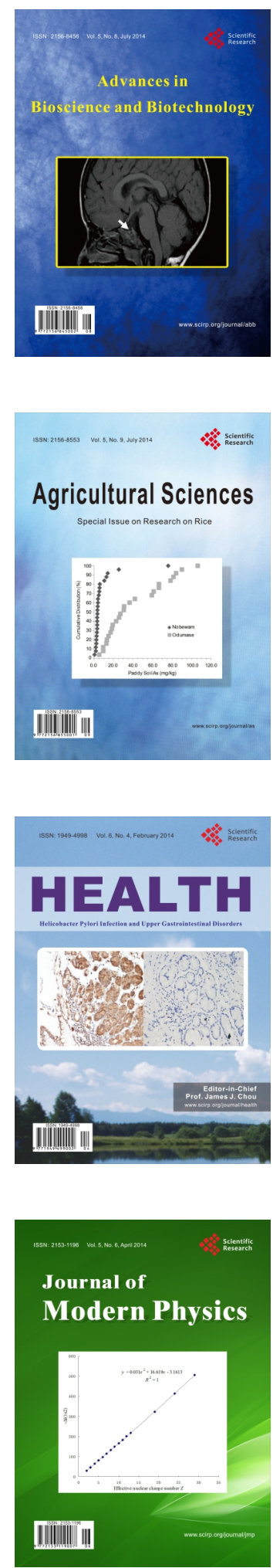
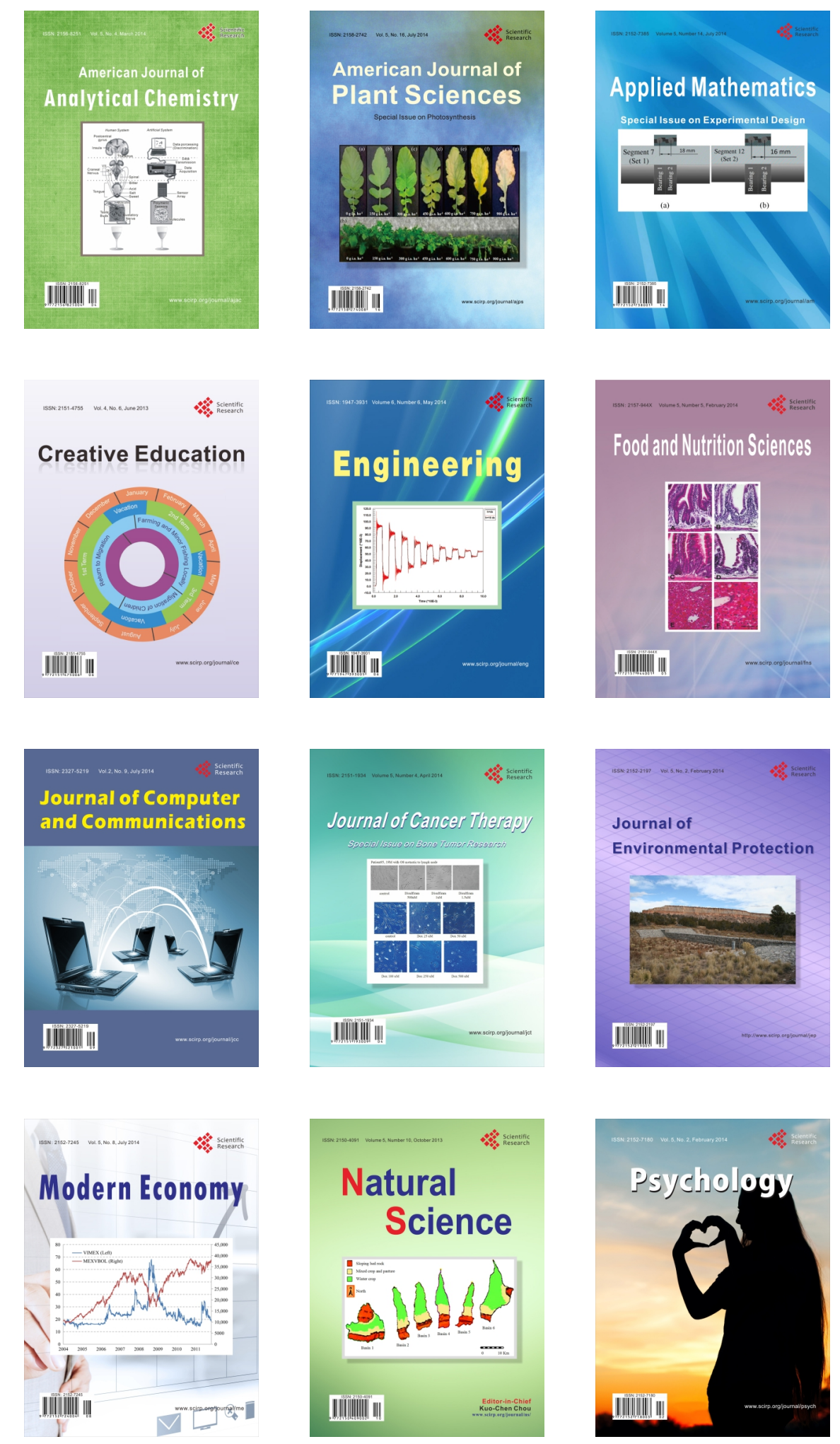